\title{
Efetividade dos Sistemas Computadorizados de Prescrição Eletrônica e de Apoio à Decisão sobre os erros de prescrição em Pediatria
}

\author{
Josiane L. B. dos Santos ${ }^{1,2}$, Sonia R. Lambert Passos ${ }^{1,2}$, Natalia S. O. Barbosa ${ }^{3}$, \\ Fernanda M. Sousa ${ }^{1}$, Luisa A. A. M. Neves ${ }^{2}$, Paula A. Motta ${ }^{2}$, Luciana T. \\ Cavalini ${ }^{3}$. \\ ${ }^{1}$ Laboratório de Epidemiologia Clínica - Instituto Nacional de Infectologia Evandro \\ Chagas/ Fundação Oswaldo Cruz \\ ${ }^{2}$ Universidade Estácio de Sá \\ ${ }^{3}$ Universidade Federal Fluminense \\ \{josiane.lima, sonia.lambert\}@ini.fiocruz.br,uff_naty@yahoo.com.br, \\ \{fernanda_m.souza,p.andrademotta\}egmail.com, \\ luisaabenathar@hotmail.com, lutricav@outlook.com
}

\begin{abstract}
Systematic review with meta-analysis, up to December 2020, in order to compare the incidence of prescription errors before and after the introduction of Electronic Prescription Systems associated or not with Decision Support Systems in different pediatric settings. In ten studies in the ICU, the reduction in dosage errors (a type of prescription error) varied from $15 \%$ to $30 \%$. Two studies presented total error suppression. In wards reductions were $36 \%, 45 \%, 67 \%$ and $74 \%$. The reduction was $7.4 \%$ in overall prescribing errors. There was clear evidence of reduction in the risk of dosing errors in pediatrics with the use of electronic prescription systems for the ICU, ward and outpatient settings.
\end{abstract}

Resumo Revisão Sistemática com metanálise, até dezembro de 2020. Objetivo comparar incidência de erros de prescrição antes e após introdução de Sistemas de Prescrição Eletrônica associados ou não a Sistemas de Apoio à Decisão em diferentes settings pediátricos. Em dez estudos em UTI a redução em erros de dosagem (um tipo de erro de prescrição) variou de $15 \%$ a $30 \%$. Dois estudos com supressão total de erros. Em enfermaria as reduções foram de 36\%, 45\%, 67\% e 74\%. A redução foi de 7,4 de erros gerais de prescrição. Evidenciou-se redução de risco de erros de dosagem em pediatria com o uso de sistemas de prescrição eletrônica para UTI, enfermaria e ambulatório.

\section{Introdução}

A prescrição médica é parte essencial do atendimento a um paciente [Nuckols et al. 2014]. Esse processo pode envolver erros, sendo eles advindos do médico ou de outro professional envolvido desde a prescrição medicamentosa, até sua dispensação e utilização pelo paciente. Qualquer falha envolvida neste processo é chamada de erro de medicação (EM). 
O EM pode trazer consequências graves e até irreversíveis para o paciente. A cada ano, inúmeros pacientes são prejudicados e cerca de um terço desses erros podem ser evitados [Ghaleb et al. 2005]. Pelas características antropométricas e fiosiológicas, como menor peso, metabolismo com menor tolerância a uma dosagem equivocada de medicamento as crianças estão sob maior risco de EM [Kazemi et al. 2011], podendo ser eles de diferentes tipo - erros de prescrição; de dosagem (superdosagem, subdose); de dispensação.

Visando facilitar o uso seguro e eficaz de medicamentos, mecanismos eletrônicos têm sido adotados pelos sistemas de saúde do mundo. Os Sistemas de Prescrição Eletrônica (SPE) e os Sistemas de Apoio à Decisão (SAD) têm demonstrado grande potencial de interferência favorável na problemática reduzindo os EM [Vélez-DíazPallarés, Pérez-Menéndez-Conde, Bermejo-Vicedo 2018] em diferentes settings adultos. Dessa forma, espera-se que possam contribuir ainda mais se utilizados na pediatria.

O objetivo deste estudo foi avaliar a incidência de erros de medicação, em pediatria, pré e pós implementação de SPE associados ou não aos SAD.

\section{Metodologia}

Trata-se de uma revisão sistemática registrada na base PROSPERO sob o número CRD 42019126590, que seguiu o Preferred reporting items for systematic reviews and metaanalyses (PRISMA statement). As buscas foram realizadas nas bases de dados PubMed, Scopus, Web of Science, ilacs, Biblioteca Cochrane, SciELO. Utilizou-se termos do MeSh e palavras chaves relacionadas à intervenção (sistema eletrônico de prescrição), desfecho (erros de prescrição) e população de estudo (pediatria; criança).

Foram incluídos estudos observacionais antes e depois da intervenção SPE com ou sem SAD sobre erros de prescrição medicamentosa em pediatria. Esses foram avaliados metodologicamente quanto a viés de seleção e classificação e perdas. Excluiuse artigos sobre sistemas diagnóstico, vacina, alimentação ou terapêuticas não medicamentosas ou não pediátricas. Extraiu-se dos incluídos as variáveis: identificação do artigo, dos autores, ano de publicação, local do estudo (atenção ambulatorial, hospitalar, de emergência, unidade de terapia intensiva), tamanho amostral (total de pacientes ou prescrições), características clínicas e sociodemográficas da população pediátrica incluída, tipo de SPE e presença ou não de SAD, tipos de erros de medicação.

Os resultados dos estudos foram avaliados em função da presença ou ausência de 6 SAD. Foram coletadas medidas de incidência e de associação (risco relativo; risco atribuível, odds ratio) com respectivos Intervalos de 95\% de confiança (IC 95\%), quando necessários foram calculadas as medidas de resumo pelas incidências descritas. A análise estatística consistiu em uma metanálise de efeitos variáveis por tipo de erro de dosagem em enfermaria, sendo elaborado um gráfico de Forest plot. Foi estudada a heterogeneidade pelo teste de $\mathrm{I}^{2}$, sendo considerada moderada acima de $50 \%$ e elevada acima de $70 \%$.

\section{Resultados}

Foram identificados 200 estudos, dos quais incluiu-se 24 nesta revisão. Publicados entre 1998 e 2018, a maioria foi realizada nos Estados Unidos da América, sendo todos na língua inglesa exceto um, em alemão. Sete foram desenvolvidos em Unidade de Terapia Intensiva (UTI), seis em enfermaria, seis em ambulatório, um em Emergência e quatro 
em cenários múltiplos. Nove estudos avaliaram os SPE exclusivamente e 15 à associação SPE e SAD.

Dos realizados na UTI, dois evidenciaram redução dos erros de medicação. Neles, relatou-se nominalmente que os erros de medicação gerais foram eliminados - passaram de $13,3 \%$ na fase pré para zero pós [Cordero et al. 2004]; ou reduziram significativamente: de 98 para 3 casos [Trotter et al. 2009]. Um deles cujo foco foi erros de dosagem também identificou eliminação dos erros pós SPE, desta vez associados aos SAD. Os erros de superdosagem eram $3,8 \%$ e de subdose eram de $9,5 \%$ pré intervenção no estudo [Cordero et al. 2004].

Quatro dos oito estudos em enfermaria informaram medidas de associação, todas com redução em erros gerais de prescrição sendo este risco 74\% menor [Kim et al. 2006]; $36 \%$ menor [Kazemi et al 2011] e 67\% menor para erros de dosagem [Sethuraman et al 2015]. Kazemi et al (2011) também descreveram redução de $45 \%$ em erros de dosagem. E finalmente, evidenciaram redução de $23 \%$ em erros de subdosagem de aminoglicosídeos [Abboud et al. 2006]. Forest Plot (Figura 1) da metanálise desses quatro estudos totalizando 19.394 prescrições revelou que incidência de erros de dosagem foi $51 \%$ menor (RR 0,49 IC 95\% (0,35 - 0,70)) após à implementação de SPE.

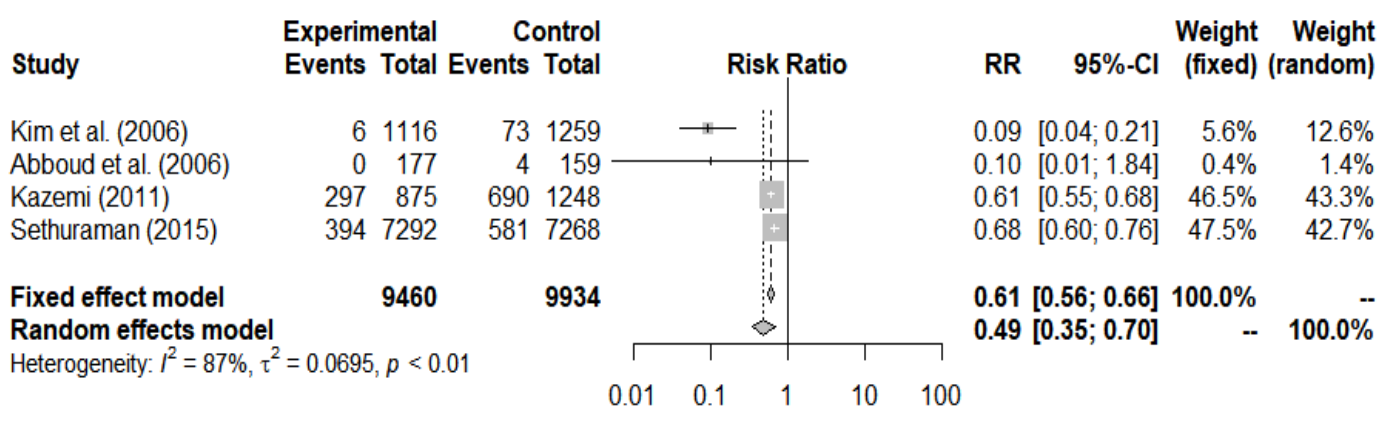

Figura 1. Forest Plot da metanálise de estudos de erros de dosagem em enfermarias pediátricas.

Todos estudos ambulatoriais apresentaram desfechos com medidas de associação. No entanto, dois estudos [Mainous et al 2013 e Litvin et al. 2013] investigaram um tipo de desfecho específico como a redução de uso inadequado de antibiótico de amplo espectro $(-19,7 \%)$ e $(-16,3 \%)$; outro: Collins et al. (2011) observou $69 \%$ de redução das chances de erro de prescrição geral de quimioterapia oral. McPhilips et al 2005 identificaram redução de $30 \%$ da chance de erros potenciais entre dois settings ambulatoriais. Em ambulatório de nefrologia pediátrica na Inglaterra [Jani et al. 2008] risco atribuível menor em 7,4\% de erros gerais de prescrição.

O único estudo realizado em emergência [Sard et al. 2008], identificou uma tendência de redução em erros de prescrição não estatisticamente significativa.

As incidências de erros de prescrição no período pré SPE, nos países desenvolvidos, foram em torno de $8 \%$ a $13,3 \%$, enquanto o estudo realizado no Irã [Kazemi et al 2011] foi de 52\%. No estudo realizado em emergência a incidência foi de $27 \%$. 


\section{Discussão e Conclusão}

Este é um tema recente na literatura médica, a qualidade dos estudos é razoavelmente boa, porém ainda faltam estudos realizados em Emergências, um setting muito propenso a erros potenciais de medicação, pela agilidade de tomada de decisões e no entanto quase não investigado. Nas duas décadas mais recentes somente um estudo publicado Sard et al. (2008), possivelmente a padronização de prescrições para quadros clínicos dinâmicos que exigem velocidade de decisão sejam ainda mais complexos. Este desafio precisa ser enfrentado nos próximos artigos.

Os resultados em enfermaria foram expressivos pós implementação de SPE, variando em reduções de pelo menos $16 \%$ à metade. Neste setting foi possível calcular medida de efeito combinada por metanálise, que indicou claramente redução de $51 \%$.

Nos settings de enfermaria pediátrica os resultados justificam plenamente a implementação de sistemas de prescrição eletrônica. Os estudos em UTI também sugeriram eliminação destes erros, sem unanimidade. Os resultados em ambulatório estão pulverizados por diagnóstico (oncologia) ou indicação (antibioticoterapia) cabendo expandir para ambulatório geral. $\mathrm{O}$ setting de emergência merece esforços de pesquisa considerando a alta frequência de erros de prescrição no período pré-implementação, de duas a três vezes a frequência de erros em países desenvolvidos.

A descrição do funcionamento e recursos de cada software poderia ser mais detalhada, bem como a opinião dos profissionais envolvidos no uso destas ferramentas poderia ser consultada durante a implementação. Estes aspectos foram estudados apenas por Baysari et al. (2018) sendo relatado o surgimento de novos erros relacionados ao emprego de SPE, por exemplo - pesos incorretos sendo usados no cálculo da dosagem e mais de uma enfermeira envolvida no preparo de uma mesma medicação. Além disso estes autores [Kadmon et al. 2009] advertiram sobre a necessidade de uma melhor adaptação inicial dos usuários com o sistema. A falta de familiaridade e de treinamento inicial provocaram opiniões contrárias ao uso do SPE.

A questão da eficiência é complicada pelo uso ao longo prazo, um estudo recente [Kadmon et al. 2017] investigou a eficiência uma década após a implementação de um sistema de prescrição eletrônica e concluiu que atualizações periódicas deveriam ser realizadas no sistema para minimizar ou evitar erros.

Embora os erros de dosagem já tenham estabelecido um paradigma de eficiência deste softwares nesta investigação outros aspectos como a interação de dois medicamentos foi abordada: Sethuraman et al (2015) demonstraram que a aplicação do SPE associado a SAD não alterou o desfecho relacionado a interação medicamentosa, sendo necessário outros métodos para resolver essa questão.

Heterogeinedade dos softwares utilizados, tempos desiguais de coleta de dados antes e depois, complexidades de doenças abrangidas pelos SAD e os protocolos aceitos pelos serviços como adequados são limitações deste estudo. Resta alguma dúvida se nesses estudos observacionais os sistemas eletrônicos foram a única mudança que modificaram o desfecho. Pontos fortes consistem não restrição de idioma, não exclusão da síntese qualitativa de estudos com diferentes tamanhos amostrais.

Houve evidências consistentes de redução de tipos de erro de medicação em pediatria com o uso de sistemas de prescrição eletrônica tanto para UTI, quanto enfermaria e ambulatório. Em contraste quase todos os esforços de pesquisa neste tema 
são ainda oriundos de países desenvolvidos. A implementação de tais sistemas parece ainda mais urgente considerando a carga em países não desenvolvidos em que a prevalência de erros pré-implementação é até seis vezes maior que nos países desenvolvidos.

\section{Referências}

Abboud P. A., Ancheta R., McKibben M., Jacobs B. (2006). Impact of workflowintegrated corollary orders on aminoglycoside monitoring in children. Health Informatics Journal. Vol 12, $\mathrm{n}^{\circ} 3$, pages 187-98.

Baysari M. T., Hardie R. A., Lake R., Richardson L., McCullagh C., Gardo A., Westbrook J. (2018). Longitudinal study of user experiences of a CPOE system in a pediatric hospital. International Journal of Medical Informatics. Vol 109, pages 5-14.

Ghaleb M. A., Barber N., Franklin B. D., Wong I. C. K. (2005). What constitutes a prescribing error in paediatrics? Quality Safety Health Care. Vol 14, $\mathrm{n}^{\circ} 5$, pages 352 7.

Kadmon G., Bron-Harlev E., Nahum E., Schiller O., Haski G., Shonfeld T. (2009). Computer order entry with limited decision support to prevent prescription errors in a PICU. Pediatrics. Vol 124, pages 935-4.

Kadmon, G., Pinchover, M., Weissbach, A., Kogan Hazan, S., Nahum, E. (2017). Case Not Closed: Prescription Errors 12 Years after Computerized Physician Order Entry Implementation. Journal of Pediatrics. Vol 190, pages 236-240.e2.

Kazemi A., Ellenius J., Pourasghar F., Tofighi S., Salehi A., Amanati A., Fors U. G. H. (2011). The effect of computerized physician order entry and decision support system on medication errors in the neonatal ward: Experiences from an Iranian teaching hospital. Jornal of Medical Systems. Vol 351, pages 25-37.

Kim G. R., Chen A. R., Arceci R. J., Mitchell S. H., Kokoszka K. M., Daniel D., Lehmann C. U. (2006). Error reduction in pediatric chemotherapy: Computerized order entry and failure modes and effects analysis. Archives of pediatrics \& adolescent medicine.

Litvin C. B., Ornstein S. M., Wessell A. M., Nemeth L. S., Nietert P. J. (2013). Use of an electronic health record clinical decision support tool to improve antibiotic prescribing for acute respiratory infections: The ABX-TRIP study. Journal of General Internal Medicine. Vol 28, $\mathrm{n}^{\circ}$ 6, pages 810-6.

Mainous A. G., Lambourne C. A., Nietert P. J. (2013). Impact of a clinical decision support system on antibiotic prescribing for acute respiratory infections in primary care: Quasi-experimental trial. Journal of America Medical Informatics Association. Vol 20, $n^{\circ} 2$, pages 317-24.

McPhillips H. A., Stille C. J., Smith D, Hecht J., Pearson J., Stull J., et al (2005). Potential medication dosing errors in outpatient pediatrics. Journal of Pediatrics.

Nuckols, T. K., Smith-Spangler C., Morton S. C., , Asch, S. M., Patel V. M., Anderson L. J., et al. (2014). The effectiveness of computerized order entry at reducing preventable adverse drug events and medication errors in hospital settings: a systematic review and meta-analysis. Systematics Reviews. Vol 3, n ${ }^{\circ} 56$. 
Sard B.E., Walsh K.E., Doros G., Hannon M., Moschetti W., Bauchner H. (2008). Retrospective evaluation of a computerized physician order entry adaptation to prevent prescribing errors in a pediatric emergency department. Pediatrics. Vol 122, pages $782-7$.

Sethuraman, U., Kannikeswaran N., Murray K. P., Zidan M. A., Chamberlain J. M (2015). Prescription Errors Before and After. Academy Emergency Medicine. Pages $714-9$.

Vélez-Díaz-Pallarés M., Pérez-Menéndez-Conde C., Bermejo-Vicedo T. (2018). Systematic review of computerized prescriber order entry and clinical decision support. American Society of Health-System Pharmacists. Vol 75, n 23.

Trotter A, Maier L. Computergestütztes Verordnungssystem bei pädiatrischen Patienten (2009). Monatsschrift Kinderheilkunde. Vol 157, pages 160-5.

Cordero L, Kuehn L, Kumar RR, Mekhjian HS (2004). Impact of computerized physician order entry on clinical practice in a newborn intensive care unit. $J$ Perinatol. Vol 24 pages 88-93. 